\title{
A constant of motion in 3D implies a local generalized Hamiltonian structure
}

\author{
Benito Hernández-Bermejo Víctor Fairén ${ }^{1}$ \\ Departamento de Física Fundamental, Universidad Nacional de Educación \\ a Distancia. Senda del Rey S/N, 28040 Madrid, Spain.
}

\begin{abstract}
We demonstrate that a Poisson structure can always be associated to a general nonautonomous 3D vector field of ODEs by means of a diffeomorphism that preserves both the orientation and the volume of phase-space. The only prerequisite is the existence of one constant of motion.
\end{abstract}

Keywords: Ordinary differential equations, generalized Hamiltonian structures, Poisson systems.

${ }^{1}$ To whom all correspondence should be addressed. E-mail vfairen@uned.es 


\section{Introduction}

The possibility of associating a generalized Hamiltonian structure [1, 2] (also known in the literature as a Poisson structure) to general flows of ODEs has deserved a considerable attention recently, both from the point of view of the existence of such representations [3] and of their explicit determination in two

[4], three $[5,6,7]$ and $n$ dimensions $[8,9,10]$. The main advantage of Poisson structures lies in that they can encompass much wider categories of flows than the classical symplectic structure (of which the Poisson structure is just a generalization). However, this is done while preserving the benefits of the classical symplectic representation, as proved by Darboux' theorem [1].

The construction of generalized Hamiltonian formulations is of interest in the field of statistical mechanics, where it has been demonstrated that Nambu mechanics [11] is just a particular case of Poisson structure [12, 13, 14]; in the Inverse Problem of Mechanics, with results which range from the classical LieKoenigs theorem for symplectic structures [15] to the relationship between Poisson structures and Birkhoff systems [16]; in the discussion of nonlinear stability of relative equilibria by the energy-Casimir method [17, 18, 19]; in evolutionary dynamics, in which the connection between Poisson structures and replicator equations for bimatrix games has been recently established by Hofbauer [20]; in quantization [21], due to the relevance of the link between Poisson and Jacobi manifolds; finally, may we mention the importance in numerical analysis where, in addition to the well-known symplectic integrators [22, 23], a new generation of algorithms is being developed [24, 25], the efficacy of which is ensured automatically if the system admits a generalized Hamiltonian description.

The Poisson structure for a system of first-order equations

$$
\dot{x}^{i}=v^{i}\left(x^{1}, \ldots, x^{n}, t\right), \quad i=1, \ldots, n
$$

is based: first, on an antisymmetric matrix $\mathcal{J}$ whose components $J^{i j}$ are, in general, functions of the $x^{i}$ and satisfy the Jacobi identities

$$
J^{l i} \partial_{l} J^{j k}+J^{l j} \partial_{l} J^{k i}+J^{l k} \partial_{l} J^{i j}=0,
$$

where $\partial_{l}$ means $\partial / \partial x^{l}$, indices $i, j, k$ run from 1 to $n$ and the summation convention over repeated indices is applied; and second, on a Hamiltonian $H$, which is usually taken to be a time-independent first integral of the system. Then the 
flow (1) is said to be Hamiltonian in the generalized sense if it can be written as

$$
\dot{x}^{i}=J^{i j} \partial_{j} H, \quad i=1, \ldots, n .
$$

In other words, $v^{i}$ can be expressed as $v^{i}=\left[x^{i}, H\right]$ for the Poisson bracket defined by

$$
[F, G] \equiv\left(\partial_{i} F\right) J^{i j}\left(\partial_{j} G\right)
$$

Of course, the task of writting an arbitrary system (1) in the form (3) cannot be accomplished in general, even if a first integral is known. This is also the case when the phase-space is three-dimensional (although this is the single dimension in which the Jacobi identities (2) are not overdetermined, as pointed out by Haas and Goedert [6]).

We shall focus this work on the three-dimensional problem. In this case, it has been demonstrated that the knowledge of two independent first integrals is always sufficient to develop a generalized Hamiltonian formulation of the problem [12]. When only one first integral is known, the present-day methods merely allow the solution of the problem in certain situations $[5,6,7]$. We shall prove here the following general result: the knowledge of a single constant of motion leads locally to a Poisson structure. Given a first integral of the flow (1), the usual approach considers a direct recasting of the system into Poisson form, thus limiting the scope of this format to just those few cases in which the procedure leading from (1) to (3) can consistently be completed. Our solution to the problem does not intend to build the Poisson structure directly on the original system (1) itself, as the previous procedures try to do. We instead overcome foregoing limitations by showing how an equivalent local Poisson structure can always be associated to an arbitrary three-dimensional system, if we first proceed to submit it to a simple orientation-preserving diffeomorphism, after which the hamiltonization procedure is straightforward. This implies automatically the strict topologically orbital equivalence between both flows [26]. Moreover, the diffeomorphism is also volume-preserving. The outcome is that it is always possible in practice to exploit the advantages derived from the Hamiltonian formulation of the problem, since both systems are dynamically equivalent and information can be directly transferred between them due to the invertibitily of the transformation. 


\section{Poisson structures in $3 \mathrm{D}$ flows}

Due to its ubiquity in all fields of Physics, Chemistry and Biology, 3D systems have received an especial attention in the literature - even an attempt of giving a sample of references about the issue would probably exceed the limits of this work. This has also been the case with regard to Poisson structures. One of the first fundamental results [12] showed that, in general, a 3D system possesses a Poisson structure if two independent first integrals exist. In a subsequent article, Gümral and Nutku [5] reduced the problem to the solution of a nonlinear partial differential equation, which can be transformed into a Riccati equation if two independent constants of motion are known. Later, Haas and Goedert [6] were able to reformulate the problem as the search of particular solutions of a linear partial differential equation. In their method (which will be our starting point) only one time-independent first integral of the system is, in principle, required; however, when two independent constants of motion are known the problem is reduced to quadrature. This approach was subsequently generalized by Goedert et al. [7] to include the case of time-dependent, rescalable first integrals. To make the list complete, we must also add those approximations which proceed directly from ansatzs for matrix $\mathcal{J}$, for the Hamiltonian or for both, such as that of Plank [27].

The result of these efforts is that it has been possible to construct a Hamiltonian formulation for some 3D systems (or, at least, for certain integrable or semi-integrable cases of them). Apart from classical examples such as Euler's equations for a free rigid body [18, 28] or a particle in a magnetic field [9], we may mention more involved (and interesting) systems like Lotka-Volterra equations [27, 29, 30], the Halphen system [5], Maxwell-Bloch equations [5], the Lorenz model [5, 7], the Rabinovich system [7], the RTW interaction [7], and also some systems of biological relevance such as the Kermac-McKendric model for epidemics [31] or the May-Leonard equations [5].

Consequently, any generalization of the previously mentioned techniques leading to the construction of Poisson structures for wider sets of flows would lead to an increase of the tools available for their analysis, as follows from the argumentation given in the Introduction. Such a generalization shall be our goal in the forthcoming sections of the paper. As mentioned before, our starting point will be the technique due to Haas and Goedert, which we briefly recall now for the sake of completeness. 
Let us consider a system of ODEs

$$
\dot{x}^{i}=v^{i}\left(x^{1}, x^{2}, x^{3}, t\right), \quad i=1,2,3,
$$

for which a time-independent constant of motion $H\left(x^{1}, x^{2}, x^{3}\right)$ exists. In short, what Haas and Goedert have shown is that, once $H$ is known, a completion of the problem is equivalent to finding one particular solution of the following linear partial differential equation, which is essentially a restatement of the Jacobi identities (2):

$$
v^{i} \partial_{i} J=A J+B
$$

where

$$
A=\partial_{i} v^{i}-\frac{\partial_{3} v^{i} \partial_{i} H}{\partial_{3} H}, B=\frac{v^{1} \partial_{3} v^{2}-v^{2} \partial_{3} v^{1}}{\partial_{3} H}
$$

Once a particular value of $J$ is known, system (5) complies to format (3) with Hamiltonian $H$ and structure matrix:

$$
J^{12}=J, J^{13}=\frac{v^{1}-J \partial_{2} H}{\partial_{3} H}, J^{23}=\frac{v^{2}+J \partial_{1} H}{\partial_{3} H}
$$

The explicit determination of a solution of (6) for an arbitrary vector field is, in principle, not guaranteed. However, as Haas and Goedert point out [6], equation (6) always admits the direct solution $J=0$ in the homogeneous case $B=0$, though they did not exploit this line of action. We shall do it here. This leads to a nontrivial solution of the Jacobi equations (2), since $J^{13}$ and $J^{23}$ do not vanish in general. Further details, such as those related to the preservation of scale invariance by equations (6) and (7) can be found in the original reference $[6]$.

\section{Construction of a Poisson structure for 3D flows}

We start by considering a system of the form (5) for which a time-independent $C^{1}$ constant of motion $I\left(x^{1}, x^{2}, x^{3}\right)$ is known:

$$
\frac{\mathrm{d} I}{\mathrm{~d} t}=v^{i} \partial_{i} I=0
$$

Thus we can write $I\left(x^{1}, x^{2}, x^{3}\right)=I_{0}$, where $I_{0}=I\left(x^{1}(0), x^{2}(0), x^{3}(0)\right)$. We shall assume without loss of generality that $\partial_{3} I \neq 0$ (if this is not the case, a 
different labeling of the variables can always be adopted in order to exchange $x^{3}$ with either $x^{1}$ or $x^{2}$, as shown by Haas and Goedert [6]).

As emphasized at the end of Section 2, the fundamental equation (6) does always admit a solution in the case $B=0$ or:

$$
v^{1} \partial_{3} v^{2}-v^{2} \partial_{3} v^{1}=0
$$

This identity holds if, in particular:

$$
\partial_{3} v^{1}=\partial_{3} v^{2}=0
$$

or, in other words, $v^{i} \equiv v^{i}\left(x^{1}, x^{2}, t\right)$ for $i=1,2$. This suggests the possibility of performing a change of dependent variables in system (5) such that (11) holds for the target system. This transformation is:

$$
\begin{aligned}
& \tilde{x}^{1}=x^{1} \\
& \tilde{x}^{2}=x^{2} \\
& \tilde{x}^{3}=I\left(x^{1}, x^{2}, x^{3}\right)+\varphi\left(x^{1}, x^{2}\right)
\end{aligned}
$$

where $\varphi\left(x^{1}, x^{2}\right)$ is an arbitrary $C^{1}$ function. The condition $\partial_{3} I \neq 0$ has two relevant implications. The first is that there exists a unique $C^{1}$ function $g$ such that the equation $I\left(x^{1}, x^{2}, x^{3}\right)=I_{0}$ can be equivalently written as:

$$
x^{3}-g\left(x^{1}, x^{2}, I_{0}\right)=0
$$

This allows the explicit inversion of the third equation in (12):

$$
x^{3}=g\left(\tilde{x}^{1}, \tilde{x}^{2}, \tilde{x}^{3}-\varphi\left(\tilde{x}^{1}, \tilde{x}^{2}\right)\right)
$$

The second implication is that (12) is one-to-one since the Jacobian is not zero. Consequently, transformation (12) is a diffeomorphism.

The equations of motion for the transformed system are then:

$$
\begin{aligned}
& \dot{\tilde{x}}^{1}=v^{1}\left(\tilde{x}^{1}, \tilde{x}^{2}, g\left(\tilde{x}^{1}, \tilde{x}^{2}, \tilde{x}^{3}-\varphi\left(\tilde{x}^{1}, \tilde{x}^{2}\right)\right), t\right) \\
& \dot{\tilde{x}}^{2}=v^{2}\left(\tilde{x}^{1}, \tilde{x}^{2}, g\left(\tilde{x}^{1}, \tilde{x}^{2}, \tilde{x}^{3}-\varphi\left(\tilde{x}^{1}, \tilde{x}^{2}\right)\right), t\right) \\
& \dot{\tilde{x}}^{3}=\dot{\tilde{x}}^{1} \tilde{\partial}_{1} \varphi\left(\tilde{x}^{1}, \tilde{x}^{2}\right)+\dot{\tilde{x}}^{2} \tilde{\partial}_{2} \varphi\left(\tilde{x}^{1}, \tilde{x}^{2}\right)
\end{aligned}
$$

where $\tilde{\partial}_{i}$ denotes $\partial / \partial \tilde{x}^{i}$. It is straightforward to check that (15) has the first integral:

$$
\tilde{x}^{3}-\varphi\left(\tilde{x}^{1}, \tilde{x}^{2}\right)=I_{0}
$$


This is, in fact, nothing else that the original constant of motion $I$ in terms of the new variables, as is evident from the third equation in (12). Then, we can re-express (15) in its final form:

$$
\begin{aligned}
& \dot{\tilde{x}}^{1}=v^{1}\left(\tilde{x}^{1}, \tilde{x}^{2}, g\left(\tilde{x}^{1}, \tilde{x}^{2}, I_{0}\right), t\right) \\
& \dot{\tilde{x}}^{2}=v^{2}\left(\tilde{x}^{1}, \tilde{x}^{2}, g\left(\tilde{x}^{1}, \tilde{x}^{2}, I_{0}\right), t\right) \\
& \dot{\tilde{x}}^{3}=\dot{\tilde{x}}^{1} \tilde{\partial}_{1} \varphi\left(\tilde{x}^{1}, \tilde{x}^{2}\right)+\dot{\tilde{x}}^{2} \tilde{\partial}_{2} \varphi\left(\tilde{x}^{1}, \tilde{x}^{2}\right)
\end{aligned}
$$

This flow verifies condition (11) and it is then a Poisson system. From (16), the Hamiltonian is $H=\tilde{x}^{3}-\varphi\left(\tilde{x}^{1}, \tilde{x}^{2}\right)$. Notice how the arbitrariness of $\varphi$ remains as an extra degree of freedom from which we can eventually profit to write the Hamiltonian in some desirable form. On the other hand, equations (8) provide the structure matrix:

$$
\mathcal{J}=\left(\begin{array}{ccc}
0 & 0 & \tilde{v}^{1}\left(\tilde{x}^{1}, \tilde{x}^{2}, t\right) \\
0 & 0 & \tilde{v}^{2}\left(\tilde{x}^{1}, \tilde{x}^{2}, t\right) \\
-\tilde{v}^{1}\left(\tilde{x}^{1}, \tilde{x}^{2}, t\right) & -\tilde{v}^{2}\left(\tilde{x}^{1}, \tilde{x}^{2}, t\right) & 0
\end{array}\right)
$$

where $\tilde{v}^{i}\left(\tilde{x}^{1}, \tilde{x}^{2}, t\right)=v^{i}\left(\tilde{x}^{1}, \tilde{x}^{2}, g\left(\tilde{x}^{1}, \tilde{x}^{2}, I_{0}\right), t\right)$ for $i=1,2$. The reduction to a generalized Hamiltonian formulation is thus achieved. Transformation (12) is actually global, not dependent on any particular value of the first integral $I$. On the contrary, the resulting equations of motion (17), and by extension the Poisson structure matrix (18), are particularized to the level surfaces of $I$, and are thus parametrized by $I_{0}$. Consequently, in order to get (17) and (18), (12) is to be applied locally, in each one of the level surfaces.

Taking into account (13), we may choose, in particular, the following representation for the constant of motion:

$$
I\left(x^{1}, x^{2}, x^{3}\right)=x^{3}-g\left(x^{1}, x^{2}, I_{0}\right)+I_{0}
$$

Once substituted into (12), the Jacobian of the transformation takes the value 1 , and it is thus volume-preserving. Since this value is positive, both the original and the target system are also topologically orbital equivalent [26].

\section{Additional considerations}

We conclude our exposition by detailing some significant particular situations not considered in the previous section. 
(I) Direct substitution of $x^{3}$ as a limit case

Let us consider transformation (12) in the case in which $I$ is written in the form (19). We can make the following choice for function $\varphi$ :

$$
\varphi\left(x^{1}, x^{2}\right)=g\left(x^{1}, x^{2}, I_{0}\right)-I_{0}
$$

Then the resulting system (17) takes the form:

$$
\begin{aligned}
& \dot{\tilde{x}}^{1}=v^{1}\left(\tilde{x}^{1}, \tilde{x}^{2}, g\left(\tilde{x}^{1}, \tilde{x}^{2}, I_{0}\right), t\right) \\
& \dot{\tilde{x}}^{2}=v^{2}\left(\tilde{x}^{1}, \tilde{x}^{2}, g\left(\tilde{x}^{1}, \tilde{x}^{2}, I_{0}\right), t\right) \\
& \dot{\tilde{x}}^{3}=\dot{\tilde{x}}^{1} \tilde{\partial}_{1} g\left(\tilde{x}^{1}, \tilde{x}^{2}, I_{0}\right)+\dot{\tilde{x}}^{2} \tilde{\partial}_{2} g\left(\tilde{x}^{1}, \tilde{x}^{2}, I_{0}\right)
\end{aligned}
$$

and the Hamiltonian is $H=\tilde{x}^{3}-g\left(\tilde{x}^{1}, \tilde{x}^{2}, I_{0}\right)+I_{0}$. Note that this system is formally the same that would result when using equation (13) to substitute directly $x^{3}$ in the original equations (5). However, no change of variables is performed when we proceed in this way. We could say, alternatively, that the final variables are just the same than the original ones. The consistency of our general approach based on a change of dependent variables can then be checked easily since equations (12) reduce, as expected, to the identical transformation when we substitute (19) and (20) in them.

(II) 2D systems without a known first integral

It is a well-known result that any $2 \mathrm{D}$ flow

$$
\dot{x}^{i}=v^{i}\left(x^{1}, x^{2}, t\right), \quad i=1,2,
$$

possessing a time-independent first integral $I\left(x^{1}, x^{2}\right)$ can be written as a Poisson system $[4,6]$. The analysis of Section 3 allows us to carry out this task when no first integral is known for equations (22) by means of a one-dimensional embedding, i.e., by recasting the flow (22) into a three-dimensional one. The standard recipe for this purpose consists of the addition of a new variable $x^{3}$ to the system. We do this in the following way:

$$
\begin{aligned}
& \dot{x}^{i}=v^{i}\left(x^{1}, x^{2}, t\right), \quad i=1,2 \\
& \dot{x}^{3}=v^{1} \partial_{1} \varphi\left(x^{1}, x^{2}\right)+v^{2} \partial_{2} \varphi\left(x^{1}, x^{2}\right)
\end{aligned}
$$

where $\varphi\left(x^{1}, x^{2}\right)$ is again an arbitrary $C^{1}$ function. System (23) is analogous to (17), and it is therefore Hamiltonian in the generalized sense with Hamiltonian 
function $H=x^{3}-\varphi\left(x^{1}, x^{2}\right)$. On the other hand, (23) is obviously equivalent to the original flow (22) in each level surface $H=$ constant.

\section{(III) Quasipolynomial systems}

Let us consider 3D systems of the quasipolynomial form:

$$
\dot{x}^{i}=x^{i}\left(M_{i 0}+\sum_{j=1}^{m} M_{i j}\left(x^{1}\right)^{B_{j 1}}\left(x^{2}\right)^{B_{j 2}}\left(x^{3}\right)^{B_{j 3}}\right), \quad i=1,2,3,
$$

where $m$ is a positive integer and the rest of coefficients are assumed to be real. These systems have proven to be suitable for representing general nonlinear flows $[32,33]$. We shall assume the existence of a first integral $I\left(x^{1}, x^{2}, x^{3}\right)$ verifying the condition $\partial_{3} I \neq 0$. If we substitute equations (24) in (10) we find:

$$
\begin{gathered}
\sum_{i, j=1}^{m}\left(M_{1 i} M_{2 j}-M_{1 j} M_{2 i}\right) B_{j 3} x_{1}^{B_{i 1}+B_{j 1}} x_{2}^{B_{i 2}+B_{j 2}} x_{3}^{B_{i 3}+B_{j 3}-1}+ \\
\quad+\sum_{k=1}^{m}\left(M_{10} M_{2 k}-M_{20} M_{1 k}\right) B_{k 3} x_{1}^{B_{k 1}} x_{2}^{B_{k 2}} x_{3}^{B_{k 3}-1}=0
\end{gathered}
$$

This leads to the nonlinear system of equations:

$$
\begin{array}{ll}
\left(M_{1 i} M_{2 j}-M_{1 j} M_{2 i}\right)\left(B_{j 3}-B_{i 3}\right)=0, & i, j=1, \ldots, m ; i \neq j \\
\left(M_{10} M_{2 k}-M_{20} M_{1 k}\right) B_{k 3}=0, & k=1, \ldots, m
\end{array}
$$

There are two possibilities:

i) There is at least one value of $i, 1 \leq i \leq m$, such that $B_{i 3} \neq 0$. Then equations (26) can be used to demonstrate that there exists a real constant $\xi$ such that $($ row2) $=\xi \times($ row1) in matrix $M$. From this it is straightforward to prove that a first integral of the form $\left(x^{1}\right)^{\xi}\left(x^{2}\right)^{-1}$ exists. Since this constant of motion verifies $\partial_{3}\left[\left(x^{1}\right)^{\xi}\left(x^{2}\right)^{-1}\right]=0$, it must be different to $I$. Then system (24) has two independent constants of motion and it is integrable.

ii) $B_{i 3}=0$ for all $i$. Then equations (26) are always satisfied.

The only nontrivial situation is therefore the second one, which implies, to sum up, that $x^{3}$ is not present in the first two equations of system (24). This is precisely the meaning of equation (11). Consequently, conditions (10) and (11) are completely equivalent, up to trivial and nongeneric cases, for quasipolynomial systems of the general form (24). 


\section{Final remarks}

In the preceding sections we have demonstrated how, for a 3D system of ODEs possessing one constant of motion, but for which a generalized Hamiltonian representation may not exist, such a structure can be achieved when the original vector field is suitably transformed to a completely equivalent system, in such a way that the information obtained (both qualitative and quantitative) from the Hamiltonian formulation can be carried back into the original flow. This approach seems to be relatively new in the literature, since the usual techniques try to build the Hamiltonian structure for the original system itself. The result is that the problem can be solved only for a limited set of ODEs or, in the case of the most general approaches, that the practical implementation of the Poisson structure is exceedingly difficult. One exception to this trend is the work of Cairó and Feix [4], where they are able to construct a symplectic structure for 2D flows by means of a rescaling of time. However, it is the authors' conjecture that additional transformations on variables apart from that on time are necessary in order to handle the problem in three and higher dimensions. In this sense, an appropriate manipulation of the phase-space variables is one of the most attractive (perhaps the unique) possibility.

\section{Acknowledgements}

This work has been supported by the DGICYT (Spain), under grant PB940390. B. H. acknowledges a doctoral fellowship from Comunidad Autónoma de Madrid. The authors also acknowledge Drs L. Cairó, F. Haas, M. Plank and G. R. W. Quispel for supplying them with copies of their works. 


\section{References}

[1] P. J. Olver, Applications of Lie Groups to Differential Equations, 2nd Ed. (Springer-Verlag, New York, 1993).

[2] A. Weinstein, J. Diff. Geom. 18 (1983) 523.

[3] V. Perlick, J. Math. Phys. 33 (1992) 599.

[4] L. Cairó and M. R. Feix, J. Phys. A: Math. Gen. 25 (1992) L1287.

[5] H. Gümral and Y. Nutku, J. Math. Phys. 34 (1993) 5691.

[6] F. Haas and J. Goedert, Phys. Lett. A 199 (1995) 173.

[7] J. Goedert, F. Haas, D. Hua, M. R. Feix and L. Cairó, J. Phys. A: Math. Gen. 27 (1994) 6495.

[8] C. A. Lucey and E. T. Newman, J. Math. Phys. 29 (1988) 2430.

[9] S. A. Hojman, J. Phys. A: Math. Gen. 24 (1991) L249.

[10] S. A. Hojman, J. Phys. A: Math. Gen. 29 (1996) 667.

[11] Y. Nambu, Phys. Rev. D 7 (1973) 2405.

[12] M. Razavy and F. J. Kennedy, Can. J. Phys. 52 (1974) 1532.

[13] G. J. Ruggeri, Int. J. Theor. Phys. 12 (1975) 287.

[14] J. A. Kobussen, Hadronic J. 1 (1978) 966.

[15] E. T. Whittaker, A Treatrise on the Analytical Dynamics of Particles and Rigid Bodies, 4th Ed. (Cambridge Univ. Press, Cambridge, 1937).

[16] R. M. Santilli, Foundations of Theoretical Mechanics, Vol. II (SpringerVerlag, New York, 1983).

[17] D. D. Holm and K. B. Wolf, Physica D 51 (1991) 189.

[18] V. I. Arnold, Ann. Inst. Fourier Grenoble 16 (1966) 319.

[19] D. D. Holm, J. E. Marsden, T. Ratiu and A. Weinstein, Phys. Rep. 123 (1985) 1. 
[20] J. Hofbauer, J. Math. Biol. 34 (1996) 675.

[21] D. Chinea, J. C. Marrero and M. de León, J. Phys. A: Math. Gen. 29 (1996) 6313.

[22] H. Yoshida, Phys. Lett. A 150 (1990) 262.

[23] M. Sanz-Serna and M. P. Calvo, Numerical Hamiltonian Problems (Chapman \& Hall, London, 1994).

[24] G. R. W. Quispel and H. W. Capel, Phys. Lett. A 218 (1996) 223.

[25] G. R. W. Quispel and G. S. Turner, J. Phys. A: Math. Gen. 29 (1996) L341.

[26] E. A. Jackson, Perspectives of Nonlinear Dynamics, Vol. 1, 1st Ed. (Cambridge University Press, Cambridge, 1994).

[27] M. Plank, J. Math. Phys. 36 (1995) 3520.

[28] E. C. G. Sudarshan and N. Mukunda, Classical Mechanics - A Modern Perspective, 2nd Ed. (Krieger, New York, 1983).

[29] M. Plank, Nonlinearity 9 (1996) 887.

[30] Y. Nutku, Phys. Lett. A 145 (1990) 27.

[31] Y. Nutku, J. Phys. A: Math. Gen. 23 (1990) L1145.

[32] E. H. Kerner, J. Math. Phys. 22 (1981) 1366.

[33] B. Hernández-Bermejo and V. Fairén, Phys. Lett. A 206 (1995) 31. 